\title{
Funcionamiento básico y social de los usuarios de las viviendas supervisadas para personas con trastorno mental severo en Asturias: necesidad de una intervención pedagógica*
}

\section{Basic and social functioning of the users of satellite housing for people with severe mental disorder in Asturias: the need for pedagogical intervention}

\author{
Omar GARCÍA-PÉREZ y Susana TORÍO LÓPEZ \\ Universidad de Oviedo
}

Recibido: Abril 2013

Aceptado: Mayo 2013

\begin{abstract}
Resumen
El proceso de reforma psiquiátrica cambió el modelo de atención a las personas con trastorno mental severo, abriendo las puertas al desarrollo de alternativas socioeducativas y de apoyo en la comunidad. La vivienda representa un elemento clave en su recuperación e inserción social, mejorando aspectos clínicos, personales y sociales. El objetivo del estudio es analizar el funcionamiento básico y social de los usuarios de las viviendas supervisadas en Asturias y evaluar la influencia del programa residencial en su perfil funcional. Se utiliza un cuestionario que recoge las variables sociodemográficas y clínicas, el Inventario de Conducta Social (SBS) y la Escala Habilidades Básicas de la Vida Diaria (BELS), mediante entrevistas a personal y usuarios de los alojamientos, quienes presentan más de cuatro disfunciones graves para la vida diaria y cuatro problemas graves de conducta. Existen diferencias en función de género, edad y el lugar de residencia previo. La estancia en el programa reduce ingresos en salud mental, aumenta la adherencia al tratamiento y mejora sus habilidades domésticas y actividades de la vida diaria, pero no sus relaciones sociales y conductuales. Así pues, se constata la necesidad de una intervención pedagógica en el ámbito comunitario, y se demuestra que la vivienda es capaz de mantener en la comunidad a personas con distintos niveles de dificultad en su conducta social, sus síntomas y sus habilidades cotidianas.
\end{abstract}

Palabras clave: Pedagogía Social; enfermedad mental; vivienda; integración social; intervención socioeducativa.

\footnotetext{
* Esta investigación ha sido subvencionada por el Gobierno del Principado de Asturias con cargo a fondos provenientes del Plan de Ciencia, Tecnología e Innovación (P.C.T.I.) de Asturias. Referencia BP06-083. 


\begin{abstract}
The psychiatric reform changed the model of care for people with severe mental illness, opening the door to the development of alternative socio and community support. Housing is a key element in their recovery and social integration, improving clinical, personal and social. The aim of the study is to analyze the basic skills of daily living and social behavior of the users in supported housing in Asturias and evaluated the influence of the residential program in their functional profile. A questionnaire was used to interview staff and users of the accommodations. Sociodemographic and clinical variables were included: the Social Behavior Inventory (SBS) and the Basic Skills Scale of Daily Living (BELS). The accommodation users had more than four serious dysfunctions in their daily living and four serious behavior problems. The results show that there are differences in terms of gender, age and previous place of residence. The housing program reduces mental health hospitalizations, increases adherence and improves their domestic skills and activities of daily living, but not their social relations and behavior. Thus, there seems to be a need for educational intervention at the community level, and housing proves to be useful to maintain people in community, even those with different levels of difficulty in their social behavior, their symptoms and daily life skills.
\end{abstract}

Keywords: Social Pedagogy; mental diseases; housing; social integration; social-educational intervention.

Durante las últimas décadas, la atención a las personas con enfermedad mental ha experimentado una profunda transformación, impulsada en España a raíz de la Reforma Psiquiátrica de los años 80. A partir de ese momento, se inicia el proceso de desinstitucionalización y se asumen el modelo de atención comunitaria como estrategia general de actuación y la rehabilitación psicosocial como filosofía de intervención (González-López, 2011; IMSERSO, 2007; Ministerio de Sanidad y Consumo, 2007 y 2011; Rodríguez, Muñoz y Panadero, 2007). Dentro de todo este nuevo paradigma de atención se sitúan las personas con trastorno mental severo (TMS), cuyo concepto no sólo incluye el diagnóstico (habitualmente esquizofrenia y otras psicosis); la duración y tratamiento (superior a dos años), sino que también se define por el funcionamiento global y la presencia de discapacidad, significando alteraciones y déficits en varios aspectos funcionales como en su conducta social (Ruggeri et al., 2000). En este sentido, tal y como expone Garcés Trullenque (2010), los problemas y necesidades de la población con enfermedades mentales desbordan el ámbito sanitario-psiquiátrico y se expresan en dimensiones de índole social, caracterizadas por discapacidades y déficits para el funcionamiento psicosocial autónomo y por consecuencias sociales de desventaja social (problemas de alojamiento, pobreza, empleo, aislamiento social, rechazo...).

Por tanto, este cambio de modelo nos sitúa ante un escenario que plantea retos y desafíos a los que es necesario dar respuestas orientadas a mejorar las realidades sociales desde una perspectiva holística. En este contexto, la Pedagogía Social debe mantener activa su capacidad para promover procesos de aprendizaje, de formación y desarrollo, con vocación de cambio y transformación social que contribuyan decisivamente al bienestar de las personas y a mejorar su calidad de vida (Caballo Villar y Gradaílle Pernas, 2008); en una permanente interrelación con el entorno y 
efectuando un proyecto socioeducativo que dé una respuesta eficaz y profesional a sus problemáticas (Del Pozo Serrano y Añaños-Bedriñana, 2013). Todo ello basado en una visión alternativa de la recuperación y el "empoderamiento" que guíe el camino hacia la inclusión y justicia social (Nelson, Aubry \& Hutchison, 2013).

De este modo, se explicita el deseo de transformar las condiciones sociales de existencia desde la sociedad, con la sociedad y para la sociedad. Asimismo, esta reconceptualización, como señala Caride $(2002,2005)$, plantea modificaciones sustanciales con relación al lugar que ocupan las personas en los procesos de acción social, reconociéndolas en su condición de agentes de cambio, con capacidad de pensar y decidir en función de sus experiencias cotidianas, siendo sujetos de la acción y no sólo objetos de atención. Por tanto, hay un nuevo giro en las demandas institucionales, intentando la Educación Social corregir la conceptualización clásica de institucionalización (Pérez Serrano, 2003) y se toma al sujeto como personas de deberes y de derechos en lo que precisamente Casas $(2006$, p.43) denomina "paradigma de los derechos", y no como un objeto de aplicación de políticas más o menos paternalistas (Núñez, 1999); de modo que la educación social, en palabras de Ortega Esteban (1999) "debe ante todo ayudar a ser y a convivir con los demás. Aprender a ser con los demás y a vivir juntos en comunidad" (p.18).

Así pues, la atención a las personas con TMS no solamente engloba la visión de la Pedagogía/Educación Social de paliar y mejorar las situaciones surgidas de la marginación y la exclusión social, sino que también sugiere la existencia, como señala Ortega Esteban (2005), de ese lado más positivo de la Pedagogía Social, que permite que la educación social muestre sus potencialidades a la hora de activar y hacer más dinámicas las condiciones educativas tanto de la cultura y de la sociedad, como de sus individuos y promueva una sociedad que eduque y una educación que socialice e integre, adoptando un modelo realmente comunitario en el marco de una educación a lo largo de la vida.

\section{Vivienda con apoyo como eje principal para inclusión social y recuperación funcional}

Dentro de este marco de actuación comunitaria, la adquisición de una vivienda digna proporciona un punto de inflexión que permite a la gente comenzar a trabajar en su recuperación y, a partir de ahí, lograr otros objetivos de vida (Ridgway, 2008). Asimismo, como indican Rogers, Kash-MacDonald \& Olschewski (2009), aparte del tratamiento, probablemente no hay área de servicios más importante para la rehabilitación y recuperación de las personas con TMS que la prestación de servicios de vivienda sobre los que ofrecer los apoyos socioeducativos necesarios de manera flexible, en función de las necesidades de sus usuarios. Esta filosofía es denominada "supported housing", sobre la que se basa el modelo de atención residencial considerado actualmente la referencia mundial gracias a la evidencia de sus resultados. Se trata del conocido "housing first" y del programa "Pathways to Housing Program" desarrollado por Tsemberis \& Eisenberg (2000) en Nueva York y que se está exportando por todo Estados Unidos y por varios países de la Unión Europea (Pleace \& 
Wallace, 2011); aun siendo un modelo reducido a la atención a los pobres y a personas sin hogar (Desviat, 2011) cuya principal motivación política para su implantación es la reducción de costos (Stanhope \& Dunn, 2011).

Por otro lado, a pesar de que diversas revisiones acerca de la literatura científica del alojamiento con apoyo para personas con TMS constatan los límites de la evidencia y la falta general de pruebas sólidas en todo el sector, como las de Chilvers, MacDonald \& Hayes, 2009; O'Malley \& Croucher, 2005 o Pleace \& Wallace, 2011, hay información suficiente para afirmar que los distintos programas residenciales se asocian con resultados positivos para las personas con TMS (Fakhoury et al., 2002; Greenwood, et al., 2005; Leff, et al., 2009; López Álvarez et al., 2004; Mares \& Rosenheck, 2004; Nelson, 2010; Nelson et al., 2013; Newman, 2001; Pleace \& Wallace, 2011; Ridgway, 2008; Rogers et al., 2009; Tsemberis, 2010):

- Son capaces de mantener en la comunidad a un número considerable de personas con trastorno mental severo.

- Ofrecen cierta estabilidad residencial.

- Mejoran el funcionamiento y la integración social.

- Reducen la incidencia de hospitalización así como los síntomas psiquiátricos.

- Aumentan el nivel de funcionamiento básico y social.

- Mejoran el nivel de vinculación con los recursos comunitarios.

- Aumentan la satisfacción con su propia vida.

- Incrementan la calidad de vida, y producen un gran sentido y pertenencia de hogar.

- Son rentables ya que reducen costes debido a un menor uso de servicios.

Así pues, la prestación de servicios residenciales emerge como uno de los pilares básicos en la recuperación e inclusión social de las personas con enfermedad mental, hasta el punto de que varios autores, como Shepherd \& Murray, sostienen que "la vivienda debe estar en el centro de la psiquiatría comunitaria" (citado por Macpherson, Shepherd, \& Edwards, 2004, p.180), a la par que un elemento clave sobre el que construir una intervención pedagógica que mejore su calidad de vida.

\section{Viviendas supervisadas para personas con TMS en el Principado de Asturias}

Aunque no existe un programa autonómico de atención residencial para personas con TMS en el Principado de Asturias, en la actualidad hay tres tipos de organización del alojamiento en viviendas supervisadas ${ }^{1}$ :

a) Aquellas cuyo apoyo se establece a través de la Fundación Asturiana de Atención y Protección a Personas con Discapacidades y/o Dependencias (FASAD). Asume la

\footnotetext{
${ }^{1}$ La información recogida en este apartado corresponde al análisis documental de los proyectos internos de las instituciones. Cabe señalar que durante el desarrollo de la investigación hubo alguna modificación en el número de viviendas y el régimen de temporalidad de las mismas, si bien los datos que se presentan en el artículo corresponden al modelo de intervención expresado en este punto.
} 
gestión de cuatro pisos distribuidos por diversas zonas del Principado de Asturias en donde residen trece personas. La finalidad de las viviendas es "ayudar a personas con trastorno mental severo a reintegrarse en la comunidad y a mejorar su funcionamiento psicosocial, de modo que les permita mantenerse en su entorno social en unas condiciones lo más normalizadas e independientes posibles". El trabajo en los pisos se enfoca desde una atención individualizada, cubriendo diferentes necesidades: alojamiento y manutención, servicios de autocuidados, actividades de la vida diaria, habilidades sociales y comunitarias, ocio y tiempo libre. Cada vivienda cuenta con el apoyo de dos cuidadores/as a tiempo parcial que cubren un horario de siete horas y media diarias de lunes a domingo. La situación de las viviendas se encuadra en entornos donde disponen de todos los servicios comunitarios a una distancia que se puede recorrer a pie. Su índice de restricción es de 16 puntos sobre un máximo de 55, configurando un espacio bastante normalizado y abierto de convivencia (García-Pérez, 2013).

b) Aquellas cuya gestión es llevada a cabo por otra institución social, Fundación Siloé, en coordinación permanente con los Servicios de Salud Mental. Fundación Siloé lleva a cabo la gestión, apoyo e intervención de dos pisos en los que conviven 6 personas, con la peculiaridad de que están situados en la misma planta de un edificio. El objetivo fundamental consiste en proporcionar al usuario un soporte psicosocial y pedagógico en su proceso de inserción, abarcando en la intervención los diferentes ámbitos vitales, hasta el logro de una estabilidad social, laboral, económica y personal que le permita la adquisición de habilidades y competencias sociales dirigidas a la consecución de una vida autónoma. Para su consecución, se desarrolla desde el equipo socioeducativo un proceso de asesoramiento y acompañamiento individualizado con un educador de referencia. La estancia es voluntaria y temporal, vinculada al proyecto de inserción social, con una cobertura de 24 horas. Asimismo, el periodo de estancia se establece de 6 meses prorrogable por periodos de seis meses hasta un máximo de 18 . Su ubicación es totalmente urbana, con todos los servicios comunitarios disponibles a su alcance. Su índice de normas restrictivas es de 17 sobre 55 puntos, lo que dan, al igual que las viviendas de FASAD, un nivel óptimo de normalización (García-Pérez, 2013).

c) Aquella cuyo apoyo depende directamente de la unidad de Rehabilitación Psicosocial o Comunidad Terapéutica. La intervención la desarrolla, de forma exclusiva, el servicio de salud mental, con personal de enfermería que realiza las visitas de supervisión, dependiente de la Comunidad Terapéutica. Por tanto, su modelo de intervención difiere de los dos explicados con anterioridad, funcionando como una estructura más del sistema sanitario, como un dispositivo intermedio de rehabilitación sin personal adscrito al piso.

Por tanto, aunque los diferentes programas residenciales ofrecen unos servicios y funciones similares, también difieren en algunas cuestiones relativas a su filosofía de trabajo y funcionamiento interno, que pueden marcar divergencias en los resultados del funcionamiento global de sus usuarios. 


\section{Método}

El objetivo general del artículo pretende analizar las habilidades para la vida diaria y conducta social de los usuarios de las viviendas supervisadas en Asturias y su relación con las características socioclínicas y el programa de atención residencial. De este modo, pretendemos dar respuesta a preguntas esenciales para el desarrollo de una intervención socioeducativa en las viviendas, con el fin de mejorar el programa y el apoyo ejercido a diario por los profesionales, de forma que revierta sobre la recuperación de estos ciudadanos y su inclusión social de una forma efectiva y no meramente terminológica: ¿Cómo es el funcionamiento global de las personas con TMS que viven en los alojamientos con apoyo de los distintos programas? ¿Cuáles son sus mayores dificultades? ¿En qué tipo de comportamientos y conducta tienen más problemas? ¿Qué características personales pueden influir en sus niveles de funcionamiento? ¿En qué es efectivo el programa residencial? ¿En qué aspectos es prioritario realizar una intervención socioeducativa? ¿Las diferencias en la gestión del programa provocan efectos diferentes en los residentes?

\section{Participantes}

Profesionales de las viviendas supervisadas. Personal trabajador de los recursos residenciales como informantes clave del funcionamiento básico y social de los usuarios.

Personas con TMS que habitan en las viviendas supervisadas. La muestra está conformada por 21 usuarios, de una población total de 22 residentes alojados, confiriendo un grado de participación del 95,45\%. Del total de la muestra, el 52,4\% son mujeres y el $47,6 \%$ hombres. La edad media de los participantes es de 42,81 $(\mathrm{DT}=8,40)$. Son solteros $(85,7 \%)$ y tienen un bajo nivel de estudios $(38,1 \%$ terminaron GB o estudios primarios; $33,3 \%$ Secundaria). Además, el $66,7 \%$ se encuentra en situación de desempleo y un $23,8 \%$ están jubilados. La mayoría de los beneficiarios del programa, un $66,6 \%$, cobra mensualmente una pensión no contributiva, y la media mensual de ingresos económicos de los residentes es de $421 €(\mathrm{DT}=230,72)$. El 52,4\% derivan de recursos de la red de Salud Mental, mientras que el 42,9\% provienen del domicilio familiar o particular.

Por otra parte, el 90,5\% presentan un diagnóstico de esquizofrenia, principalmente, paranoide. La edad media de inicio de la enfermedad es de 23,7 años $(\mathrm{DT}=7,37)$. Han sufrido una media de 3,11 ingresos por usuario $(\mathrm{DT}=2,35)$ en servicios de salud mental. Sumado a ello, el $95 \%$ han de seguir tratamiento farmacológico y llevan en el alojamiento una media de 30 meses (DT=30,27), si bien el 28,6\% lleva menos de seis meses, el $23,8 \%$ entre seis meses y un año, el $19 \%$ de uno a dos años y el $28,6 \%$ más de dos años.

\section{Instrumentos}

Habilidades Básicas de la Vida Diaria (BELS-Basic Everyday Living Schedule) (Grupo Andaluz de Investigación en Salud Mental, 2000). Instrumento diseñado para 
evaluar las habilidades específicas básicas de convivencia diaria en personas que sufren un trastorno mental de larga duración (Casas et al., 2010). Se administra a un informante clave, en este caso el personal de las viviendas y explora cuatro áreas fundamentales de funcionamiento: autocuidado, habilidades domésticas, habilidades comunitarias, y actividad y relaciones sociales. El cuestionario se compone de 26 ítems en un escala de cinco niveles para el desempeño de las habilidades: nivel normal de realización (4) problemas menores (3 puntos), problema moderado o necesidad ocasional de una sugerencia ( 2 puntos), problema serio o necesidad frecuente de una sugerencia ( 1 punto), no realiza o es necesaria una supervisión diaria ( 0 puntos).

En España existe un estudio de fiabilidad realizado con una muestra de 77 usuarios de pisos y casas-hogar protegidas, cuyos resultados muestran, en general, una concordancia global muy buena (valor medio de 0.743) (Jiménez et al., 2000).

Inventario de Conducta Social (SBS- Social Behaviour Schedule) (Wykes \& Sturt, 1986). El inventario cuenta con 21 ítems, que evalúan la capacidad del sujeto para relacionarse con los demás, la adecuación de su comportamiento social y la adaptación a las exigencias del medio donde vive, mediante una escala tipo Likert, de cero (problema severo y frecuencia diaria) a cuatro (no existe conducta problemática). La información se refiere a su conducta durante el último mes a partir de la información obtenida en una entrevista semiestructurada con un informador clave, el personal de la vivienda. Su puntuación máxima es de 78 , representando no tener ningún tipo de problema o alteración comportamental. Pueden obtenerse dos tipos de puntuaciones: problemas de conducta leves y moderados (número de ítems con una puntuación igual o superior a 2) y problemas de conducta graves y muy graves (número de ítems con una puntuación inferior a 2). El SBS ha sido adaptado a la población española por Salvador-Carulla et al. (1998), cuya evaluación de fiabilidad test-retest alcanzó un porcentaje de acuerdo del $80 \%$ y valores kappa adecuados para la mayoría de los ítems de la escala.

\section{Procedimiento}

La técnica de recogida de información utilizada fue la entrevista, cuya administración se realizó de forma individual en las propias viviendas supervisadas en las que residía la muestra objeto de estudio, tras la obtención de los permisos necesarios de todos los agentes implicados en la investigación y del consentimiento informado por parte de los participantes. Su aplicación fue llevada a cabo por un único entrevistador entrenado para tal fin, con el objetivo de evitar posibles desviaciones en las interpretaciones.

\section{Variables y análisis}

Se muestra, en primer lugar, un estudio descriptivo del funcionamiento en habilidades de la vida diaria y conducta social y se identifican las disfunciones y problemáticas más graves para una posterior intervención. En segundo lugar, se realiza 
un estudio correlacional con el fin de establecer diferencias significativas que expliquen los problemas funcionales en base al género, edad, nivel educativo, lugar de residencia anterior y aspectos clínicos. Finalmente, se analizan los resultados en función de variables clave (gestión, índice ambiental, tiempo que llevan residiendo en el alojamiento, número de ingresos hospitalarios desde que residen en los pisos y las mejoras percibidas por los usuarios) respecto al programa residencial que determinen la posible influencia de las viviendas en su funcionamiento.

Se efectúan los análisis descriptivos de las variables objeto de estudio. Posteriormente, se evalúan las relaciones y diferencias de funcionamiento entre los ciudadanos alojados en las viviendas con el objetivo de esclarecer las posibles causas. Debido al tamaño de la muestra $(\mathrm{n}<30$ personas) y que la mayor parte de variables se encuentran fuera de la curva de normalidad, se emplean las técnicas no paramétricas $\mathrm{H}$ de Kruskal-Wallis y U de Mann Whitney y, en el caso de correlaciones bivariadas, el estadístico de Spearman. El tratamiento de los datos se llevó a cabo a través del programa informático estadístico Statistical Package For Social Sciences (SPSS), versión 15.0.

\section{Resultados}

En el presente apartado se expondrán los principales resultados del estudio, en donde se muestra, por un lado, cuál es el funcionamiento básico de los residentes de las viviendas $\mathrm{y}$, por otro, su funcionamiento social. Posteriormente se presenta su funcionamiento en base a las características demográficas de los usuarios y, finalmente, los resultados obtenidos del análisis de la incidencia de las viviendas en sus habilidades de la vida diaria y conducta social.

\section{Funcionamiento básico de los residentes}

El área de autocuidados de la escala de desempeño de BELS es la que obtiene una valoración más alta, por lo que los habitantes de las viviendas desempeñan notablemente estas habilidades. En este sentido, suelen ser muy independientes en la mayor parte de las cuestiones (con puntuaciones cercanas al 4, que representa el nivel normal de realización), necesitando mayor supervisión y apoyo en aspectos como la higiene personal, ya que los profesionales de las viviendas han de recordar ocasionalmente que se afeiten o se cepillen los dientes. En el otro extremo está la puntuación media que los residentes consiguen en el bloque de actividad y relaciones sociales (con una puntuación que supera escasamente el 2 de media), de forma que se relacionan con pocas personas conocidas, no buscan compañía ni ayuda en situaciones de emergencia y prefieren actividades de ocio más individuales que grupales, mostrando períodos frecuentes de inactividad. Esto demuestra una disfunción más palpable en el desarrollo de las habilidades que componen el constructo, siendo evidente el severo problema que tienen en la variable de sociabilidad (Tabla 1). 


\begin{tabular}{|c|c|c|c|c|c|}
\hline \multirow[t]{2}{*}{ Habilidades Básicas de la Vida Diaria } & \multicolumn{5}{|c|}{$\begin{array}{l}\text { Puntuación de los usuarios }(4=\text { nivel de } \\
\text { realización normal/independencia) }\end{array}$} \\
\hline & $\mathbf{N}$ & Media & DT & Asimetría & Curtosis \\
\hline Independencia movimientos & 21 & 3,33 & 1,06 & $-1,303$ & 2,254 \\
\hline Despertarse y levantarse & 21 & 2,76 & 1,09 &,- 753 & ,493 \\
\hline Vestirse & 21 & 3,33 & 0,85 &,- 743 & $-1,220$ \\
\hline Vuelta a casa por la noche & 21 & 4 & 1,09 & 4,583 & 21,000 \\
\hline Comidas & 21 & 3,43 & 0,81 & $-1,613$ & 2,821 \\
\hline Medicación & 21 & 2,62 & 1,02 &, 572 & $-1,402$ \\
\hline Higiene personal & 21 & 2,29 & 1,34 &,- 172 & $-1,195$ \\
\hline Ropa & 21 & 2,76 & 1,30 &,- 718 &,- 762 \\
\hline Incontinencia & 21 & 3,86 & 0,47 & $-3,530$ & 12,578 \\
\hline Comportamiento en el cuarto de baño & 21 & 3,62 & 0,66 & $-1,588$ & 1,428 \\
\hline AUTOCUIDADOS & 21 & 3,22 & 0,36 &,- 404 &,- 121 \\
\hline Preparación de comidas & 21 & 2,38 & 0,80 &,- 844 &,- 865 \\
\hline Preparación de comidas simples & 21 & 3,71 & 0,71 & $-3,101$ & 10,581 \\
\hline Compra de comestibles & 21 & 2,43 & 1,36 &, 035 & $-1,930$ \\
\hline Compras & 21 & 2,81 & 1,43 &,- 640 & $-1,303$ \\
\hline Lavado de ropa & 21 & 3,48 & 0,92 & $-1,581$ & 1,317 \\
\hline Cuidado de espacio personal & 21 & 2,43 & 1,36 &,- 096 & $-1,493$ \\
\hline Cuidado de su casa o zonas comunes & 21 & 2,76 & 1,04 &,- 055 & $-1,326$ \\
\hline "HABILIDADES DOMÉSTICAS & 21 & 2,86 & 0,70 & 2,044 & $-1,390$ \\
\hline "Uso de transporte público & 21 & 3 & 1,34 & $-1,098$ & "244 \\
\hline Uso de servicios asistenciales & 21 & 2,81 & 1,03 &,- 794 &,- 312 \\
\hline Uso de los locales y servicios públicos & 21 & 3,14 & 1,06 & $-1,417$ & 2,313 \\
\hline capacidad para controlar su presupuesto & 21 & 2,86 & 1,35 &,- 520 & $-1,666$ \\
\hline $\begin{array}{l}\text { HABILIDADES COMUNITARIAS } \\
\end{array}$ & 21 & 2,95 & 0,82 &,- 997 & 072 \\
\hline "Ocupación diaria & 21 & 2,43 & 1,20 &,- 192 &,- 879 \\
\hline Actividades de tiempo libre & 21 & 2,14 & 1,15 & ,345 &,- 513 \\
\hline Sociabilidad & 21 & 1,86 & 1,31 &, 143 & $-1,381$ \\
\hline Consideración por otros & 21 & 2,14 & 1,55 &, 002 & $-1,505$ \\
\hline Prestar auxilio en una emergencia & 21 & 2,81 & 1,36 & $-1,187$ &, 471 \\
\hline $\begin{array}{c}\text { ACTIVIDAD Y RELACIONES } \\
\text { SOCIALES } \\
\end{array}$ & 21 & 2,28 & 0,88 & 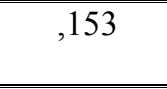 &,- 582 \\
\hline $\begin{array}{l}\text { NÚMERO DE DISFUNCIONES } \\
\text { GRAVES }\end{array}$ & 21 & 4,48 & 3,12 & 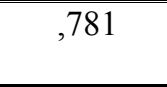 & 2,073 \\
\hline
\end{tabular}

Tabla 1.- Estadísticos Descriptivos Habilidades Básicas de la Vida Diaria.

En cuanto a las habilidades domésticas y comunitarias, su valor promedio está cercano a 3 (2,86 el primero y 2,95 el segundo), representando unos valores más altos que en otros estudios de las mismas características (Lascorz, et al., 2009; Rickard et al., 2002). En este sentido, aunque en ocasiones necesiten de supervisión o de sugerencias ocasionales, mantienen un buen grado de independencia, si bien tienen algunas dificultades para el lavado de ropa, el cuidado del espacio personal y general 
de la vivienda, lo que provoca, en ocasiones, conflictos de convivencia con sus compañeros y compañeras de piso y el personal laboral del mismo.

Por otra parte, el número de disfunciones graves entre los residentes es variable, llegando a tener una persona doce habilidades cuyo desempeño es gravemente deficitario, aunque el grupo mayoritario de residentes se encuadra en la categoría de entre una y tres disfunciones graves $(47,6 \%)$ (Tabla 2).

\begin{tabular}{|c|c|}
\hline \multirow[t]{2}{*}{$\mathrm{N}^{0}$ de problemas graves } & Vivienda supervisada \\
\hline & Usuarios con disfunciones graves \\
\hline Ninguno & $0 \%$ \\
\hline De 1 a 3 & $47,6 \%$ \\
\hline De 4 a 7 & $42,9 \%$ \\
\hline Más de 7 & $9,5 \%$ \\
\hline Media & 4,43 disfunciones graves por usuario \\
\hline
\end{tabular}

Tabla 2.- Número de disfunciones graves de los usuarios de las viviendas supervisadas.

\section{Funcionamiento Social}

La puntuación media es de 60,67, lo que confiere un buen nivel general de funcionamiento social. Son recurrentes los problemas de concentración $(0,86)$, depresión $(2,05)$, demanda de atención $(2,57)$ de las personas con trastorno mental severo, así como la proliferación de problemas en otras conductas no específicas de la escala, como alteraciones del sueño, excesos en la comida o algún tipo de trastorno obsesivo-compulsivo asociado (Tabla 3).

\begin{tabular}{l||ccccc}
\hline \multicolumn{1}{c||}{ Conductas Funcionamiento Social } & \multicolumn{5}{c}{$\begin{array}{c}\text { Puntuación de los usuarios } \\
\text { (4=nivel de realización normal/ningún problema de } \\
\text { conducta) }\end{array}$} \\
\hline Comunicación espontánea & N & Media & DT & Asimetría & Curtosis \\
\hline Lenguaje incoherente & 21 & 2,95 & 1,203 & $-1,423$ & 1,779 \\
Conversación inapropiada & 21 & 3,24 & 1,375 & $-1,622$ & 1,305 \\
Relación social inapropiada & 21 & 3,24 &, 944 & $-2,105$ & 6,317 \\
Hostilidad & 21 & 3,14 & 1,276 & $-1,250$ &, 359 \\
Demanda de atención & 21 & 3,38 &, 921 & $-1,313$ &, 726 \\
Ideas o conductas suicidas & 21 & 2,57 & 1,720 &,- 688 & $-1,403$ \\
Ataques de pánico o fobia & 21 & 3,71 &, 784 & $-2,867$ & 7,821 \\
Hiperactividad e inquietud & 21 & 3,24 & 1,091 & $-1,033$ &,- 460 \\
Reír o hablar a solas & 21 & 3,00 & 1,183 &,- 801 &,- 890 \\
Realización de ideas bizarras & 21 & 3,19 & 1,167 & $-1,450$ & 1,510 \\
Posturas y manierismos & 21 & 1,76 &, 539 & $-2,318$ & 5,058 \\
Hábitos socialmente inaceptables & 21 & 3,48 &, 928 & $-1,581$ & 1,317 \\
Conducta destructiva & 21 & 3,33 & 1,111 & $-1,470$ &, 705 \\
& 21 & 2,90 &, 301 & $-2,975$ & 7,562 \\
\hline
\end{tabular}




\begin{tabular}{l|ccccc}
\cline { 2 - 5 } Depresión & 21 & 2,05 & 1,071 &,- 642 &,- 975 \\
Conducta sexual inapropiada & 21 & 3,57 &, 746 & $-1,464$ &, 652 \\
Apariencia personal e higiene & 21 & 2,57 & 1,165 &,- 295 & $-1,386$ \\
Lentitud & 21 & 2,90 & 1,044 &,- 958 & 1,336 \\
Nivel de actividad & 21 & 2,67 & 1,354 &,- 524 & $-1,200$ \\
Concentración & 21 & 0,86 &, 727 &, 229 &,- 945 \\
\hline Inventario de Conducta Social & 21 & 60,67 & 11,101 &,- 277 &,- 966 \\
Problemas de conducta grave & 21 & 3,76 & 2,844 &, 670 &,- 127 \\
\hline
\end{tabular}

Tabla 3.- Estadísticos descriptivos Inventario de Conducta Social.

Sumado a ello, encontramos diferencias en número de conductas disruptivas o problemas graves que tienen los usuarios, habiendo quienes no presentan complicación severa y otros con 10 actuaciones graves de las analizadas, si bien la mayor parte oscilan entre tres y cinco comportamientos problemáticos (Tabla 4).

\begin{tabular}{|c|c|}
\hline \multirow[t]{2}{*}{$\mathbf{N}^{0}$ de problemas } & Vivienda supervisada \\
\hline & Usuarios con problemas Graves-Muy graves \\
\hline Ninguno & $9,5 \%$ \\
\hline De 1 a 2 & $28,5 \%$ \\
\hline De 3 a 5 & $42,8 \%$ \\
\hline Más de 5 & $15,2 \%$ \\
\hline Media & 3,76 problemas graves de conducta \\
\hline
\end{tabular}

Tabla 4.- Número de conductas graves de los usuarios.

Funcionamiento básico y social en función de las características socioclínicas

En cuanto al género, se constata que no hay diferencias significativas en ninguna de las áreas de habilidades que integran el funcionamiento básico cotidiano, a excepción del desempeño en su higiene personal, de modo que son los hombres quienes realizan la limpieza y cuidado personal de un modo más apropiado que las mujeres $(1,73<2,90$; $\mathrm{U}=26,5 ; \mathrm{p}=0,040$ ). Por otra parte, también las mujeres que residen en las viviendas supervisadas son más hostiles que los hombres en las relaciones con su entorno, ya que obtienen peor puntuación en la escala $(2,91<3,90 ; \mathrm{U}=23,5 ; \mathrm{p}=0,11)$; tienen mayores problemas de depresión $(1,55<2,60 ; \mathrm{U}=25,5 ; \mathrm{p}=0,026)$; un mayor número de problemas graves de conducta $(5,18>2,20 ; \mathrm{U}=89 ; \mathrm{p}=0,016)$ y un funcionamiento social global claramente inferior $(55<66,90 ; \mathrm{U}=21,5 ; \mathrm{p}=0,018)$.

Respecto a la edad, a medida que estas personas se hacen mayores, aumenta su desempeño en la dimensión de habilidades domésticas $(\mathrm{rs}=0,46 ; \mathrm{p}=0,037)$, realizando un mejor cuidado de su espacio personal $(\mathrm{rs}=0,55 ; \mathrm{p}=0,010)$ y del espacio común $(\mathrm{rs}=0,46 ; \mathrm{p}=0,038)$. De igual forma, los usuarios más jóvenes tienen mayores problemas de hostilidad ( $\mathrm{rs}=0,47 ; \mathrm{p}=0,034)$; más frecuentes ataques de pánico o fobia $(\mathrm{rs}=0,48 ; \mathrm{p}=0,027)$; peor apariencia personal e higiene $(\mathrm{rs}=52 ; \mathrm{p}=0,015)$; una conducta social global más deficitaria $(\mathrm{rs}=0,46 ; \mathrm{p}=0,034)$ y mayor número de problemas graves de comportamiento $(\mathrm{rs}=-0,58 ; \mathrm{p}=0,006)$. 
El nivel educativo es un componente que no influye en la forma de comportarse de $o$ en sus habilidades para la vida diaria $(\mathrm{p}>0,05)$.

Otra variable que puede influir en el funcionamiento global de los residentes, y que deben tener en cuenta los programas de intervención, es el lugar de residencia previo. En este sentido, las personas que provienen del domicilio familiar, en comparación con aquellas que vivían en Comunidad Terapéutica, tienen mayores habilidades comunicativas $(U=6 ; p=0,000)$; y obtienen mejores puntuaciones en la dimensión de actividad y relaciones sociales del BELS $(\mathrm{U}=15,5 ; \mathrm{p}=0,005)$, destacando una mayor sociabilidad $(U=4,5 ; p=0,000)$; una mejor independencia de movimientos $(U=20$; $\mathrm{p}=0,003)$ y de ocupación de actividades y tiempo libre $\sin$ apoyo $(\mathrm{U}=22,5 ; \mathrm{p}=0,016)$.

Respecto al funcionamiento básico y funcionamiento social en función de variables clínicas, como el diagnóstico y la edad de comienzo de la enfermedad, indicar que ninguna de ellas presenta correlación significativa.

\section{Incidencia de las viviendas supervisadas en el funcionamiento global de sus usuarios}

En este epígrafe se analizará la influencia del alojamiento en aspectos clínicos como el número de ingresos hospitalarios consecuencia del TMS y la adherencia al tratamiento. Asimismo, se evalúa el funcionamiento global de los residentes en base a diversas variables que pueden determinar la efectividad de los programas y las diferencias entre los mismos: por un lado la gestión de las viviendas y el tipo de programa residencial que llevan a cabo, el índice de restricción de los alojamientos, el tiempo que llevan viviendo bajo en estos recursos y sus preferencias de vivienda, así como sus valoraciones acerca de las mejoras percibidas desde que convive en los alojamientos.

En primer lugar, se constata que las hospitalizaciones a causa del TMS se han visto reducidas considerablemente desde que residen en las viviendas supervisadas de forma que 17 de las 21 personas que componen la muestra no han tenido que ser hospitalizados en la unidad de psiquiatría. Además la media de ingresos ha descendido de más de tres $(3,11)$ a un menos de uno por persona $(0,20)$.

Por otra parte, el hecho de estar en una vivienda supervisada mejora la adherencia al tratamiento, de modo que antes de la entrada al alojamiento, un $23,8 \%$ de quienes hacen uso de los alojamientos no tomaba la medicación prescrita, siendo actualmente la adherencia del tratamiento de un $100 \%$. En este sentido, el personal ha de supervisar la toma de medicación por parte como requisito indispensable para la permanencia en el alojamiento.

En cuanto a la gestión y desarrollo de los distintos programas, existen diferencias funcionales de sus usuarios en el desempeño de las habilidades domésticas $\left(\chi^{2}=9,34\right.$; $\mathrm{p}=0,009)$; en el promedio de actividades y relaciones sociales $\left(\chi^{2}=6,87 ; \mathrm{p}=0,032\right)$; en el número de habilidades de la vida diaria con graves dificultades para su desempeño $\left(\chi^{2}=7,84 ; p=0,020\right)$; independencia en el uso de servicios sociales y asistenciales 
$\left(\chi^{2}=7,33 ; p=0,026\right)$ del cuestionario BELS; y en la apariencia e higiene del SBS $\left(\chi^{2}=8,91 ; 0,012\right)$. En todos los casos, realizada la comparación por parejas, las personas del piso que gestiona directamente la Comunidad Terapéutica, son más independientes en todas las categorías y tienen menos problemas que los residentes bajo gestión de Siloé y FASAD.

Otra característica de las viviendas, que enlaza directamente con la gestión de los programas, es el nivel de restricción o ambiente interno de los alojamientos. En este sentido, no existen diferencias significativas en el funcionamiento normativo de los pisos y tampoco se constata diferencia alguna con el funcionamiento básico y social de sus residentes.

Por otro lado, el tiempo que llevan en el programa residencial tiene una relación significativa con la reducción del número de ingresos hospitalarios $(\mathrm{rs}=-0,50$; $\mathrm{p}=0,027$ ), así como se constata el hecho de que cuanto más tiempo conviven en los alojamientos, presentan un menor número de disfunciones graves en las actividades de la vida diaria $(\mathrm{rs}=-0,51 ; \mathrm{p}=0,018)$. De igual forma, relaciona de forma positiva con la el desempeño de habilidades domésticas, mejorando según transcurre el tiempo en la vivienda ( $r s=0,57 ; p=0,007)$, así como en la compra de comestibles ( $r s=0,68 ; p=0,001)$; la consideración y preocupación por los demás ( $\mathrm{rs}=0,62 ; \mathrm{p}=0,003)$; y en la reducción de la hiperactividad e inquietud ( $\mathrm{rs}=0,43 ; \mathrm{p}=0,001)$.

Las preferencias del usuario a la hora de dónde les gustaría vivir no influyen sobre su perfil funcional, de modo que no hay relación entre el hecho de no desear cambiar de residencia o desee vivir solo, o con pareja y familia respecto a su nivel de desempeño en las habilidades de la vida diaria o su conducta social.

Por último, todos los usuarios manifiestan en las entrevistas que el estar en el programa les ha ayudado, con distintos niveles de intensidad, pero consideran que han "aprendido a hacer bastantes cosas; antes no hacia la compra y ahora tengo que administrar el dinero que hay para comprar para todos en el piso" (U18). Otra persona nos indicaba que "aunque no me gusten cosas sé que si quiero vivir solo tengo que saber organizarme, no gastar dinero en tonterías y relacionarme con la gente porque luego me siento mejor" (U1). Sumado a ello, más de la mitad de los residentes ha experimentado, según sus palabras, cambios a mejor en relación a su enfermedad y casi el 70\% manifiesta una mejoría general en sus vidas desde que viven en estos pisos, dándoles "estabilidad, tengo más independencia y tengo que gente con la que sé que puedo hablar y me van a entender, y eso siempre ayuda". (U15). "Sé que si necesito algo o tengo algún problema, alguien va a echarme una mano y eso hace que no me agobie tanto...no sé, es como si una cosa llevara a otra, aunque un día me enfade y me entre la locura, tengo más seguridad" (U4), nos comentaba otra usuaria. Otros, en cambio, planteaban sus dudas en cuanto a su forma de actuar porque "sé que esto es una oportunidad para aprender a ser independiente, o eso me dicen, la quiero aprovechar, que no me echen del piso, porque si lo hago bien luego podré vivir solo y no habrá gente todo el día vigilando, aunque me apoyarán si lo necesito” (U19). 


\section{Discusión y conclusiones}

En cuanto al funcionamiento básico, los mayores problemas son las habilidades concernientes a la dimensión de actividad y relaciones sociales, destacando, por encima del resto, la baja puntuación obtenida para los niveles de sociabilidad. En general, presentan mejores habilidades para la vida diaria que otros estudios de similares características, tanto españoles como europeos (López Álvarez et al.,, 2005; Maestro et al., 2001; Rickard et al., 2002), aunque, de manera más general, tienen un perfil parecido al de otras poblaciones de programas con las mismas características (Besora, Martorell y Clusa, 2000; Fisher et al., 2001; Leff et al., 2000; Lelliot et al., 1996).

Respecto al funcionamiento social de los residentes, éstos tienen una media de casi cuatro problemas graves de conducta, siendo los más característicos la depresión, la demanda de atención y las dificultades para concentrarse. En este sentido, los usuarios de los programas residenciales en Asturias tienen unos problemas sociales ligeramente más graves que otros programas del ámbito nacional, como el de FAISEM en Andalucía, en los que sus residentes en viviendas supervisadas solamente presentan 1 problema de comportamiento severo (López Álvarez et al., 2005). Sin embargo, tal y como ocurría en el funcionamiento básico, los perfiles de los participantes del programa son similares a los presentados en otros estudios (López Álvarez et al., 2005; Maestro et al., 2001; Rickard et al., 2002; Vázquez Morejón y Jiménez García-Bóveda, 1994).

Estos parámetros pueden estar dentro de la "normalidad" si tenemos en cuenta los perfiles funcionales generales de las personas con TMS. Sin embargo, los programas han de hacer especial hincapié en la intervención en sus habilidades sociales y el apoyo para mejorar sus conductas y establecer relaciones, que generen una red de apoyo social fuera de la vivienda, poniendo a la comunidad y su entorno próximo como campo para la mejora y recuperación de esas necesidades. Además, dentro de la variabilidad de situaciones, hay un pequeño grupo de residentes con un número bastante elevado de disfunciones tanto en su funcionamiento básico como en el social, en lo que el Team for the Assessment of Psychiatric Services (TAPS) denomina "pacientes dificiles de situar" (Trieman \& Leff, 1996, p.290). Sin embargo, se demuestra en la investigación que incluso las personas con discapacidades graves son capaces de vivir en la comunidad con los apoyos necesarios (Ridgway, 2008), ofreciendo resultados positivos, de modo que las viviendas supervisadas aumentan la estabilidad residencial, fundamental para iniciar el proceso de inclusión social (Tsemberis \& Eisenberg, 2000).

El género, la edad y el lugar de residencia previo son componentes diferenciales que se deben tener en cuenta en las viviendas supervisadas. Las mujeres presentan un mayor número de problemas graves de comportamiento y un funcionamiento social más deficitario que los hombres, hecho que justifica el mayor número de mujeres en los programas residenciales, al contrario de lo que sucede en otros programas de alojamiento como Andalucía o Castilla-La Mancha. Además, según un estudio de Jiménez García-Bóveda y Vázquez Morejón (2006), las mujeres con esquizofrenia 
suelen tener una evolución más favorable y un mejor funcionamiento social, algo que no ocurre en las viviendas supervisadas asturianas y que debe ser un elemento de intervención a tener en consideración.

La edad también juega un papel importante en el funcionamiento básico y social por cuanto los más jóvenes son quienes presentan mayores problemáticas. En este sentido, nos encontramos ante un nuevo perfil de usuarios determinado, en gran medida, por el cierre y desaparición de los hospitales psiquiátricos (Fakhoury, et al., 2002; López Álvarez et al., 2005; Ogilvie, 1997). Esta nueva tipología la forman personas habitualmente más jóvenes, que nunca han estado internados en un hospital psiquiátrico, con más dificultades de adaptación y potencialmente más exigentes, al asumir un rol bastante más activo que el tradicional (López Álvarez et al., 2004). Por tanto, se constata la necesidad de realizar no sólo la detección temprana de posibles trastornos sino también iniciando las intervenciones y apoyos adecuados en la comunidad y en el ámbito escolar, con el fin de que no pierdan su proyecto vital.

Respecto a la incidencia que pueda tener el programa residencial en los habitantes de las viviendas supervisadas, se constata su efectividad para ofrecer estabilidad residencial y para la mejora de las actividades de la vida diaria (Pleace \& Wallace, 2011), pero no para su mejora social, debido a que las habilidades para realizar compras, cuestiones de aseo, limpieza, etc. son elementos básicos que se trabajan desde el primer día en los programas y es una rutina diaria que transcurre en el quehacer cotidiano. Sin embargo, se debe poner hincapié en las actividades sociales, más allá de compañeros de piso o del personal de la vivienda, para fomentar dichas conductas y su inserción social. Los programas han de promover mayores opciones de elección y control de vivienda a sus usuarios, variables asociadas a un mejor funcionamiento independiente, a una mayor estabilidad residencial y psicológica y a un mayor funcionamiento en la comunidad, tal y como Nelson (2010) concluye, cuestionando la idea de que son los profesionales quienes saben lo que es mejor para las personas enfermas.

Las diferencias de resultados funcionales desde el punto de vista estadístico entre los programas residenciales que se desarrollan en Asturias son escasas, ya que las características del piso, las intervenciones y el apoyo que se presta suelen ser similares, adaptando cuestiones que han resultado positivas en otros programas como el dormitorio individual, que mejora su funcionamiennto (Nelson, Hall \& Walsh-Bowers, 1998); viviendas con un número reducido de residentes y el hecho de convivir con otras personas con TMS hacen que funcionen mejor y obtengan resultados positivos (Rogers et al., 2009). Así pues, no es tan importante la gestión de los programas como el tiempo que llevan en el alojamiento y que enlaza con la pretendida estabilidad residencial, tal y como expresan Newman y Goldman (2008) en su escrito titulado "Putting housing first, making housing last", de forma que la vivienda sea el punto de partida para conseguir el cambio de rol de "cliente" a un papel de ciudadano por medio de la vivienda, así como mediante la educación y las oportunidades de trabajo (Piat \& Sabetti, 2011). Así pues, los usuarios sienten que mejoran, que tienen un lugar donde vivir que demuestra efectos positivos en relación a la enfermedad (Greenwood et al., 2005; Mares \& Rosenheck, 2004; Nelson et al., 2013; Ridgway. 2008; Rogers et al., 
2009), reduciendo los ingresos psiquiátricos y mejorando la adherencia al tratamiento. Además, resulta de gran importancia el hecho de que las propias personas perciben mejoría, incrementando su autoestima y su autoconfianza para la realización de cualquier actividad, con la seguridad de tener una vivienda y los apoyos necesarios para su realización.

Sin embargo, aun cuando los alojamientos mejoran aspectos clínicos y habilidades de la vida diaria, hay que poner la atención al posible peligro de institucionalización, puesto que, en gran medida, el círculo de apoyo social queda reducido al personal del alojamiento y a los compañeros de piso, creando un microcosmos que reduce su participación comunitaria (García-Pérez, 2013). Por tanto, los programas residenciales han de dar un paso más, no sólo en número de recursos, sino de concepto y desarrollo, ofreciendo el apoyo educativo, formativo y laboral necesarios en vida independiente, puesto que como ya demostraron Segal \& Kotler en 1993 en un estudio de 10 años de seguimiento, se puede producir una reducción del funcionamiento social y un aumento de la actividad social asistida, haciéndose más dependientes a la vivienda supervisada (citado en Nelson, 2010). Así pues, han de enlazar realmente con lo comunitario, ya que, como comenta Desviat (2011): "lo comunitario no es la rotación por los centros de salud mental [...], ni un programa de atención a los pobres, como ha quedado reducido en Estados Unidos [...], lo comunitario es el trabajo en red, es la acción en un territorio en continua interacción con sus ciudadanos y sus organizaciones. Una ciudadanía que forma parte del proceso, que hace suyo el proceso asistencial" (p.292).

Por este motivo se hace pertinente no solamente un cambio paliativo, sino un cambio transformador, siguiendo la propuesta de Nelson (2010), de modo que las personas con problemas de salud mental no sólo deban estar en la comunidad, sino que deben ser miembros valiosos de la comunidad, de tal forma que las políticas sociales aseguren la justicia social y una asignación de recursos más equitativa, garantizando una amplia gama de servicios alternativos de vivienda como un derecho básico de la vida, más allá de un medio terapéutico.

Se trata de conseguir un entorno comunitario favorable a la aceptación de las discapacidades que la enfermedad comporta y propiciar una optimización del uso de recursos disponibles en la comunidad, y en esto los profesionales socioeducativos tenemos una función primordial. Sin embargo, estas actuaciones de inclusión social en la comunidad no son posibles si realmente las personas con TMS no viven en la comunidad de manera efectiva. Por tanto, facilitar el alojamiento es el primer elemento básico de intervención sobre el que actuar, ofreciendo, en primer lugar, una estabilidad residencial acorde con la elección y preferencias de los usuarios y, a partir de ahí y en función de las necesidades y demandas individuales, establecer los apoyos vitales, educativos, laborales, sociales y de ocio adecuados que generen una mejora en su funcionamiento básico y social.

Así pues, la acción socioeducativa en la comunidad debe superar las limitaciones de la intervención clínica, centrada en el individuo y de carácter más reparador (Barriga, 2006), de modo que tal y como March Cerdá señalaba ya en 1988, "debe huirse de las 
soluciones y tratamientos parciales, e ir a acciones globales y holísticas" (p.89), tratando de implementar acciones educativas de una manera coordinada y en comunidad que incida en los aspectos básicos de sus necesidades con programas de apoyo educativo (de mantenimiento y acceso a insituciones formales educativas); de apoyo formativo-ocupacional y de inserción laboral; ocio y tiempo libre; educación para la salud; en el área de convivencia, familiar y de pareja; autonomía en el entorno comunitario y desenvolvimiento en el medio. Asimismo, es necesario eliminar el estigma y degradación social que sufren las personas con TMS. Todo ello con el objetivo en mente de la recuperación de su proyecto vital y no solamente la consideración paliativa de evitar la exclusión social. Sin embargo, para el logro de estas acciones es imprescindible trabajar en y con la comunidad, en y con el barrio, el entorno, la ciudad, así como disponer de las políticas sociales y recursos adecuados que prioricen el bienestar y calidad de vida de sus ciudadanos.

Por todo ello, se dibuja un espacio importante para desarrollar intervenciones de apoyo comunitario que intenten mejorar su funcionamiento global, en donde la labor pedagógica tiene un ámbito de especial relevancia.

\section{Limitaciones del estudio}

Las principales limitaciones del estudio residen en que no genera evidencia científica debido a las características metodológicas del estudio y su escasa muestra, no efectuando un procedimiento experimental, un ensayo aleatorio que muestre claramente que las mejoras percibidas en los usuarios tienen como causa directa su residencia en las viviendas supervisadas. Sin embargo, al igual que Nelson et al. (2010) o Rogers et al. (2009), consideramos de gran relevancia mostrar el estado actual de la cuestión puesto que se trata de una investigación que busca la utilidad para gestores, profesionales, trabajadores y participantes de los programas, máxime siendo un trabajo necesario como así se transmitió desde los servicios de Salud Mental del Principado de Asturias.

\section{Referencias bibliográficas}

BARRIGA, S. (2006). Cambio Social e Intervención Comunitaria. En $\mathrm{M}^{\mathrm{a}} \mathrm{I}$. Hombrados Mendieta, M. A. García Martín y T. López Espigares (coords.), Intervención Social y Comunitaria, 17-25. Málaga: Ediciones Algibe.

BESORA, J., MARTORELL, B. y CLUSA, J. (2000). Evaluación de resultados de un programa de pisos para trastornos mentales severos. Informaciones Psiquiátricas, 159, 163-173.

CABALLO VILLAR, B. y GRADAÍlLE PERNAS, R. (2008). La educación social como práctica mediadora en las relaciones escuela-comunidad local. Pedagogía Social. Revista Interuniversitaria, 15, 45-55. 
CARIDE, J.A. (2002). La Pedagogía Social en España. En V. Núñez (coord). La educación en tiempos de incertidumbre: las apuestas de la Pedagogía Social, 81112. Barcelona: Editorial Gedisa.

CARIDE, J.A. (2005). Las fronteras de la Pedagogía Social. Barcelona: Editorial Gedisa.

CASAS, E. et al. (2010). Instrumentos de evaluación en rehabilitación psicosocial. Revista de la Asociación Española de Neuropsiquiatría., XXX (105), 25-47.

CASAS, F. (2006). Bienestar y calidad de vida. En M ${ }^{\text {a }}$ I. Hombrados Mendieta, M. A. García Martín y T. López Espigares (coords.), Intervención Social y Comunitaria, 27-52. Málaga: Ediciones Algibe.

CHILVERS, R., MACDONALD, G \& HAYES, A. (2009). Supported housing for people with severe mental disorders. Cochrane Schizophrenia Group. DOI: 10.1002/14651858.CD000453.pub2

DEL POZO SERRANO, F.J. y AÑAÑOS-BEDRIÑANA, F.T. (2013). La Educación Social Penitenciaria: ¿de dónde venimos y hacia dónde vamos? Revista Complutense de Educación, 24 (1), 47-68.

DESVIAT, M. (2011). Panorama actual de las políticas de bienestar y la reforma psiquiátrica en España. Estudos de Psicologia, 16 (3), 289-294.

FAKHOURY, W. et al. (2002). Research in supporting housing. Social Psychiatry and Psychiatric Epidemiology, 37, 301-315.

FISHER, W. H. et al. (2001). Long-Stay Patients in State Psychiatric Hospitals at the End of the 20th Century. Psychiatric Services, 52 (8), 1051-1056.

GARCÉS TRULLENQUE, E.M ${ }^{\mathrm{a}}$ (2010) El Trabajo Social en Salud Mental. Cuadernos de Trabajo Social, 23, 333-352.

GARCÍA-PÉREZ, O. (2013). Viviendas supervisadas para personas con trastorno mental severo en Asturias: ¿ambiente restrictivo o abiertas a la comunidad? Pedagogía Social. Revista Interuniversitaria, 22, 123-136.

GONZÁLEZ-LÓPEZ, A.C. (coord.) (2011). Plan de Salud Mental del Principado de Asturias 2011-2016. Oviedo: Gobierno del Principado de Asturias.

GREENWOOD, R.M. et al. (2005). Decreasing psychiatric symptoms by increasing choice in services for adults with histories of homelessness. American Journal of Community Psychology, 36, 223-238.

GRUPO ANDALUZ DE INVESTIGACIÓN EN SALUD MENTAL (2000). BELS. Habilidades Básicas de la Vida Diaria. Granada: Grupo Andaluz de Investigación en Salud Mental.

IMSERSO. (2007). Modelo de Atención a Personas con Enfermedad Mental Grave. Colección Documentos: Serie de Documentos Técnicos. Madrid: Ministerio de Trabajo y Asuntos Sociales. 
JIMÉNEZ, J.F. et al. (2000). Evaluación del funcionamiento de la vida diaria en personas con trastorno mental de larga evolución. Adaptación y fiabilidad de la versión española del "Basic Everyday Living Skills" (BELS). Actas Españolas de Psiquiatría, 28 (5), 284-288.

JIMÉNEZ GARCÍA-BÓVEDA, R. y VÁZQUEZ MOREJÓN, A.J. (2006). Esquizofrenia y género. Apuntes de Psicología, 24 (1-3), 157-183.

LASCORZ, D. et al. (2009). Estudio comparativo coste-eficiencia en un dispositivo residencial para enfermos con trastorno mental severo. Revista AEN, XXIX (103), 191-201.

LEFF, J. et al. (2000). The TAPS Project: A report on 13 years of research, 1985-1998. Psychiatric Bulletin, 24, 165-168.

LELLIOT, P. et al. (1996). The mental health residential care study: classification of facilities and description of residents. British Journal of Psychiatry, 169, 139-147.

LÓPEZ ÁlVAREZ, M. et al. (2004). Los programas residenciales para personas con trastorno mental severo. Revisión y propuestas. Archivos de Psiquiatría, 67 (2), 101-128.

LÓPEZ ÁlVAREZ, M. et al. (2005). Evaluación del Programa Residencial para personas con trastorno mental severo en Andalucía (IV): perfiles funcionales y redes sociales de los residentes. Rehabilitación Psicosocial, 2 (2), 44-55.

MACPHERSON, R., SHEPHERD, G. \& EDWARDS, T.R. (2004). Supported accommodation for people with severe mental illness: a review. Advances in Psychiatric Treatment, 10, 180-188.

MAESTRO, J.C. et al. (2001). Estudio de las alternativas residenciales para pacientes desinstitucionalizados en Granada y Sevilla. Anales de Psiquiatría, 17 (4), 143-152.

MARCH CERDÁ, M. X. (1988). La intervención pedagógico-social en el ámbito de la inadaptación social: hacia una pedagogía de la inadaptación social. Pedagogía Social. Revista Interuniversitaria, 3, 81-100.

MARES, A.S. \& ROSENHECK, R.A. (2004). One-Year Housing Arrangements Among Homeless Adults With Serious Mental Illness in the ACCESS Program. Psychiatric Services, 55 (5), 566-574.

MINISTERIO DE SANIDAD Y CONSUMO (2007). Estrategia en Salud Mental del Sistema Nacional de Salud, 2006. Madrid: Ministerio de Sanidad y Consumo.

MINISTERIO DE SANIDAD Y CONSUMO (2011). Estrategia en Salud Mental del Sistema Nacional de Salud, 2009. Madrid: Ministerio de Sanidad y Consumo.

NELSON, G., AUBRY, T. \& HUTCHISON, J. (2013). Housing and Mental Health. En J.H. Stone \& M. Blouin, (eds.) International Encyclopedia of Rehabilitation. Recuperado de http://cirrie.buffalo.edu/encyclopedia/en/article/132/ 
NELSON, G. (2010). Housing for people with serious mental illness: approaches, evidence, and transformative change. Journal of Sociology \& Social Welfare, XXXVII (4), 123-146.

NELSON, G., HALL, G.B. \& WALSH-BOWERS, R. (1998). The relationship between housing characteristics, emotional well-being, and the personal empowerment of psychiatric consumer/survivors. Community Mental Health Journal, 34, 57-69.

NEWMAN, S. (2001). Housing attributes and serious mental illness: implications for research and practice. Psychiatric Services, 52, 1309-1317.

NEWMAN, S. \& GOLDMAN, H. (2008). Putting Housing First, Making Housing Last: Housing Policy for Persons with Severe Mental Illness. American Journal of Psychiatry, 165 (10), 1242-1248.

NÚÑEZ, V. (1999). Pedagogía Social: cartas para navegar en el nuevo milenio. Buenos Aires: Santillana.

OGILVIE, R.J. (1997). The state of supported housing for mental health consumers: A literature review. Psychiatric Rehabilitation Journal, 21 (2), 122-131.

O'MALLEY, L. \& CROUCHER, K. (2005). Supported housing services for people with mental health problems. Housing Studies, 20 (5), 831-845.

ORTEGA ESTEBAN, J. (1999). Educación social especializada. Concepto y profesión. En J. Ortega Esteban (coord.), Educación Social Especializada, 13-41. Barcelona: Editorial Ariel.

ORTEGA ESTEBAN, J. (2005). Pedagogía Social y Pedagogía Escolar: la Educación Social en la Escuela. Revista de Educación,336, 111-127.

PÉREZ SERRANO, G. (2003). Pedagogía Social. Educación Social. Construcción científica e intervención práctica. Madrid: Narcea Ediciones.

PIAT, M. \& SABETTI, J. (2011). Residential Housing for Persons with Serious Mental Illness: The Fifty Year Experience with Foster Homes in Canada. En J.H. Stone \& M. Blouin, (eds.). International Encyclopedia of Rehabilitation. Recuperado de http://cirrie.buffalo.edu/encyclopedia/en/article/236/.

PLEACE, N. \& WALLACE, A. (2011) Demonstrating the Effectiveness of Housing Support Services for People with Mental Health Problems: A Review. London: National Housing Federation.

RICKARD, C. et al. (2002). Residential care for mentally ill people in Andalusia and London - a comparison of care environments, users' attitudes and cost of care. Journal of Mental Health, 11 (3), 327-337.

RIDGWAY, P. (2008). Supported Housing. En K.T. Mueser \& D.V. Jester (eds.). Clinical handbook of schizophrenia, 287-298. New York: The Gilford Press. 
RODRÍGUEZ, A., MUÑOZ, M. y PANADERO, S. (2007). Descripción de una red recursos de atención social para personas con enfermedad mental grave y crónica: el caso de la Comunidad de Madrid. Rehabilitación Psicosocial, 4 (1), 41-49.

ROGERS, E. S., KASH-MACDONALD, M. \& OLSCHEWSKI, A. (2009). Systematic Review of Supported Housing Literature, 1993 - 2008. Boston: Boston University, Sargent College, Center for Psychiatric Rehabilitation. Recuperado de http://www.bu.edu/drrk/research-syntheses/psychiatric-disabilities/supportedhousing/

RUGGERI, M. et al. (2000). Definition and prevalence of severe and persistent mental illness. British Journal of Psychiatry, 177, 149-155.

SALVADOR L. et al. (1998). A reliability study of the Spanish version of the Social Behaviour Schedule (SBS) in a population of adults with learning disabilities. Journal of Intellectual Disability Research, 42(1), 22-28.

SPSS. (2006). SPSS for Windows (Version 15.0). Chicago: SPSS Inc.

STANHOPE, V. \& DUNN, K. (2011). The curious case of Housing First: The limits of evidence based policy. International Journal of Law and Psychiatry, 34, .275-282. Doi: 10.1016/j.ijlp.2011.07.006.

TRIEMAN, N., LEFF, J. (1996). The TAPS project 36: the most difficult to place long-stay psychiatric in-patients outcome one year after relocation. British Journal of Psychiatry, 169, 289-92.

TSEMBERIS, S. \& EISENBERG, R.F. (2000). Pathways to housing: Supported housing for street-dwelling homeless individuals. Psychiatric Services, 51, 487-493.

TSEMBERIS, S. (2010). Housing First: ending homelessness, promoting recovery and reducing costs. En I. Gould Ellen \& B. O'Flaherty (eds.). How to House the Homeless. 37-57. New York: Russell Sage Foundation.

VÁZQUEZ MOREJÓN, A.J. y JIMÉNEZ GARCÍA-BÓVEDA, R. (1994). Inventario de Conducta Social (Social Behaviour Schedule): fiabilidad y validez de un instrumento de evaluación para pacientes mentales crónicos. Actas Luso-Españolas de Neurología Psiquiátrica, 22 (1), 34-39.

WYKES, T. \& STURT, E. (1986). The Measurement of Social Behaviour in Psychiatric Patients: An Assessment of the Reliability and Validity of the SBS Schedule. British Journal of Psychiatry, 148, 1-11.

\section{Correspondencia con los autores}

Omar GARCÍA-PÉREZ

Universidad de Oviedo

Facultad de Formación del Profesorado y Educación

C/Aniceto Sela, s/n.

33005 Oviedo, Asturias.

e-mail: garciaomar@uniovi.es 